\title{
Holography for Heavy Quarks and Mesons at Finite Chemical Potential
}

\author{
Carlo Ewerz*, Ling Lin, Andreas Samberg, Konrad Schade \\ Institut für Theoretische Physik, Universität Heidelberg, \\ Philosophenweg 16, 69120 Heidelberg, Germany \\ and \\ ExtreMe Matter Institute EMMI, GSI Helmholtzzentrum für Schwerionenforschung \\ Planckstraße 1, 64291 Darmstadt, Germany \\ E-mail: C.Ewerz@thphys . uni-heidelberg.de, \\ L. Lin@thphys . uni-heidelberg.de, \\ A. Samberg@thphys.uni-heidelberg.de, \\ K.Schadedthphys.uni-heidelberg.de
}

\begin{abstract}
We study the properties of heavy quarks as probes of strongly coupled plasmas with and without chemical potential by means of the gauge/gravity (AdS/CFT) duality. We compute the screening distance of a heavy quark-antiquark pair, its free energy, and the running coupling in large classes of non-conformal models arising as deformations of pure AdS space. We further investigate the energy loss of a heavy quark moving on a circular orbit as an example of an accelerated motion. These observables exhibit universal features independent of the deformation, pointing to strongcoupling universality. Our results should be relevant for processes involving heavy quarks and their bound states in the quark-gluon plasma, including the case of finite net baryon density.
\end{abstract}

9th International Workshop on Critical Point and Onset of Deconfinement - CPOD2014,

17-21 November 2014

ZiF (Center of Interdisciplinary Research), University of Bielefeld, Germany

* Speaker. 


\section{Introduction}

The AdS/CFT (or gauge/gravity) duality [1,2,3] offers the possibility to address a variety of questions concerning strongly coupled systems in a holographic setup. One of the most interesting applications of the duality is the study of the strongly coupled quark-gluon plasma produced in heavy-ion collisions at RHIC and LHC, for recent reviews see for example [4, 5]. While static properties of the quark-gluon plasma can be well described in lattice gauge theory, dynamical phenomena at strong coupling pose a more difficult challenge for which the AdS/CFT duality is particularly well suited.

Originally, the AdS/CFT duality was found to relate the dynamics of maximally supersymmetric $\mathrm{SU}\left(N_{c}\right)$ Yang-Mills theory (SYM) in the large- $N_{c}$ limit and at strong coupling to weakly coupled gravity on 5-dimensional Anti-de Sitter $\left(\mathrm{AdS}_{5}\right)$ space. Loosely speaking, one can think of the gauge theory as living on the 4-dimensional boundary of the 5-dimensional AdS space, that is at $z=0$ where $z$ denotes the additional holographic (bulk) coordinate. A finite temperature of the gauge theory is on the gravity side of the duality represented by a black-hole horizon in the bulk located at a point $z_{h}$ in the fifth coordinate and extended in the other 4 space-time directions. In addition, a chemical potential of the gauge theory can be described in this setup by giving the black hole an electric charge.

Maximally supersymmetric Yang-Mills theory, a scale invariant or conformal field theory (hence the name CFT) differs strongly from QCD. The applicability of the AdS/CFT duality to real-world processes is therefore far from obvious. However, at finite temperature the two theories appear less different: On the one hand, QCD enters a deconfined phase without chiral symmetry breaking above the critical temperature and many observables become almost scale invariant. In SYM theory, on the other hand, supersymmetry and conformal symmetry are broken at finite temperature. But this is not really sufficient for making reliable predictions based on the AdS/CFT duality. More promising is the construction of dual gravity theories in which conformal symmetry is broken from the outset. This requires to introduce some sort of deformation of the AdS space of the original duality as we will describe in more detail below. In one important line of research one tries to construct theories of this type which model many properties of QCD as precisely as possible, see for example [6], although an exact gravity dual of QCD seems prohibitively difficult to find in this way. We follow a different approach here by looking at large classes of deformations of AdS space. It turns out that some observables exhibit a remarkable universality in large classes of theories, either by having a fixed value independent of the deformation, or by changing only very little or consistently in one direction as the theory is deformed. The most prominent example of such an observable is the ratio $\eta / s$ of shear viscosity to entropy density which assumes the value $1 /(4 \pi)$ in many theories obtained as duals of deformed AdS spaces [7, 8]. Universal properties of this kind might be a sign of universal strong-coupling dynamics that is more or less independent of the underlying microscopic theory. If indeed universal in this sense, such behavior would have a good chance to be very relevant for the study of the quark-gluon plasma explored in experiments.

\section{Holographic Models}

Let us now discuss in more detail the relevant AdS spaces and their non-conformal deforma- 
tions which describe strongly coupled theories at finite temperature and at finite chemical potential.

\subsection{Finite Temperature}

According to the AdS/CFT correspondence, $\mathscr{N}=4$ supersymmetric Yang-Mills theory at finite temperature is dual to gravity on $\mathrm{AdS}_{5}$ with a Schwarzschild black hole at a position $z_{h}$ in the bulk,

$$
\mathrm{d} s^{2}=\frac{L_{\text {AdS }}^{2}}{z^{2}}\left[-f(z) \mathrm{d} t^{2}+\mathrm{d} \vec{x}^{2}+\frac{\mathrm{d} z^{2}}{f(z)}\right]
$$

where

$$
f(z)=1-\frac{z^{4}}{z_{h}^{4}}
$$

$L_{\mathrm{AdS}}$, the AdS curvature radius, is assumed to be large which implies a large 't Hooft coupling of the gauge theory. The temperature of the gauge theory coincides with the Hawking temperature of the black hole and is given by $T=\left(\pi z_{h}\right)^{-1}$.

Theories closer to actual QCD can be obtained by deforming the AdS metric. One possibility is to modify the above metric in an ad-hoc way by introducing a simple factor which breaks conformal invariance. In [9] and [10] such a deformation was proposed in which the metric (2.1) is multiplied by an overall $z$-dependent factor $\exp \left(c z^{2}\right)$ with a dimensionful parameter $c$. The relation between temperature and the position of the horizon, $T=\left(\pi z_{h}\right)^{-1}$, is not changed. The resulting model is a finite-temperature version of the popular soft-wall model at $T=0$ [11], and we hence denote it by $\mathrm{SW}_{T}$. This model allows to obtain many interesting quantities in simple calculations, often even analytically. But the model does not solve the equations of motion of any 5-dimensional gravitational Einstein-Hilbert action. As a consequence, its consistency is questionable. For example, one does not expect it to lead to consistent thermodynamics in the dual 4-dimensional theory. The model also gives rise to unphysical solutions for certain string configurations corresponding to the motion of a heavy quark through the plasma in the dual theory [12].

A more promising way to make closer contact with the quark-gluon plasma is to add further fields to the 5-dimensional gravity, and then to obtain a non-conformal metric as a solution of a gravitational action. In the simplest case one adds only one scalar field $\Phi$ as proposed in [13, 14], considering the Einstein-Hilbert action

$$
S_{\mathrm{EHs}}=\frac{1}{16 \pi G_{\mathrm{N}}^{(5)}} \int \mathrm{d}^{5} x \sqrt{-G}\left(\mathscr{R}-\frac{1}{2}(\partial \Phi)^{2}-V(\Phi)\right),
$$

where $G$ is the determinant of the deformed metric $G^{\mu v}, G_{\mathrm{N}}^{(5)}$ is the 5-dimensional Newton constant, $\mathscr{R}$ is the Ricci scalar, and $V(\Phi)$ is a potential for the scalar. We are interested in classes of theories in which the metric has the general form

$$
\mathrm{d} s^{2}=\mathrm{e}^{2 A(z)}\left(-h(z) \mathrm{d} t^{2}+\mathrm{d} \vec{x}^{2}\right)+\mathrm{e}^{2 B(z)} \frac{\mathrm{d} z^{2}}{h(z)} .
$$

The resulting temperature of the dual theory is in this case obtained as

$$
T=\mathrm{e}^{A\left(z_{h}\right)-B\left(z_{h}\right)} \frac{\left|h^{\prime}\left(z_{h}\right)\right|}{4 \pi},
$$


with $z_{h}$ the position of the black hole horizon, that is $h\left(z_{h}\right)=0$. The case of the finite-temperature AdS metric (2.1) is recovered for $A(z)=B(z)=0$ and $h(z)=f(z)$. Metrics obtained as solutions of a 5-dimensional Einstein-Hilbert action are consistent in the sense that they will lead to consistent thermodynamic relations in the dual theory. At the same time, the holographic calculation of observables becomes more complicated if the metric to be used first needs to be obtained - usually numerically - from the equations of motion of (2.4).

The construction chosen here gives us a class of bottom-up models for plasmas hopefully close to that of QCD. In such bottom-up models, we have no a priori constraint on the nature of the scalar field $\Phi$. In particular, $\Phi(z)$ can be the dilaton but can also be some other type of scalar field. The dilaton typically gives a contribution in the action for a string moving on the metric $G^{\mu \nu}$, while other scalars will not affect the string in that way. We decide to consider both possibilities, taking them as independent models. As in [14] we call the corresponding models 'string frame' and 'Einstein frame' model, respectively.

The action (2.3) allows one to demand solutions resembling models of soft-wall type. Specifically, we can make the ansatz

$$
\mathrm{d} s^{2}=\mathrm{e}^{2 A(\Phi)}\left(-h(\Phi) \mathrm{d} t^{2}+\mathrm{d} \vec{x}^{2}\right)+\mathrm{e}^{2 B(\Phi)} \frac{\mathrm{d} \Phi^{2}}{h(\Phi)},
$$

where we have used a gauge that identifies the squared holographic coordinate with the scalar $\Phi$. Further choosing

$$
A(\Phi)=\frac{1}{2} \log \left(\frac{L_{\mathrm{AdS}}^{2}}{z^{2}}\right)-\frac{1}{2} c z^{2}, \quad \Phi=\sqrt{\frac{3}{2}} \phi z^{2}
$$

we can solve the equations of motion of (2.3) for $B(z)$, the horizon function $h(z)$, and the potential $V(\Phi)$. To calculate a suitable potential $V(\Phi)$ required for a soft-wall type metric might seem unusual from a top-down perspective. In our bottom-up approach the main requirement is to have a physically interesting class of consistent models, while a particular form of the potential $V(\Phi)$ is not necessary. As a result of our procedure we have a class of consistent deformed models with two independent parameters, $c$ and $\alpha \equiv c / \phi$, cf. [14]. Their temperature is obtained via (2.5) from the metric functions at the horizon, that is at the zero of $h$.

\subsection{Finite Temperature and Finite Chemical Potential}

In the framework of the AdS/CFT duality, $\mathscr{N}=4$ SYM theory with a chemical potential is obtained by making the black hole in the holographic dimension charged. The corresponding metric is an $A d S_{5}$ space with a Reissner-Nordström black hole (AdS-RN). It is given by the metric

$$
\mathrm{d} s^{2}=\frac{L_{\mathrm{AdS}}^{2}}{z^{2}}\left[-h(z) \mathrm{d} t^{2}+\mathrm{d} \vec{x}^{2}+\frac{\mathrm{d} z^{2}}{h(z)}\right],
$$

where now

$$
h(z)=1-\left(1+Q^{2}\right)\left(\frac{z}{z_{h}}\right)^{4}+Q^{2}\left(\frac{z}{z_{h}}\right)^{6} .
$$

The temperature of the boundary theory is given in terms of the charge $Q$ of the black hole by $T=\left(1-\frac{1}{2} Q^{2}\right) /\left(\pi z_{h}\right)$. The chemical potential is $\mu=\sqrt{3} Q / z_{h}$, and $Q$ is in the range $0 \leq Q \leq \sqrt{2}$. 
We should point out that the chemical potential implemented in this way is not the quark (or baryon) chemical potential of QCD but a chemical potential corresponding to the $R$-charge of $\mathscr{N}=4 \mathrm{SYM}$ theory. However, in this context it can serve as a simple way of introducing finite density effects into the system.

Also in the case of finite chemical potential one can introduce an explicit breaking of conformal invariance in order to arrive at a dual theory closer to QCD. The simplest way of doing so is again to introduce by hand an overall warp factor motivated by the soft-wall model [11]. The corresponding model, obtained by multiplying the metric (2.8) by $\exp \left(\tilde{c}^{2} z^{2}\right)$, has been proposed and studied in [15]. As discussed above, such ad hoc models allow one to get a rough picture of the effects of non-conformality, but because they do not minimize any gravitational action they suffer from inconsistencies.

Consistent non-conformal metrics dual to theories with chemical potential can be found by adding a $\mathrm{U}(1)$ field $A_{\mu}$ to the 5-dimensional gravitational action (2.3), resulting in the EinsteinHilbert-Maxwell-scalar action

$$
S_{\mathrm{EHMs}}=\frac{1}{16 \pi G_{\mathrm{N}}^{(5)}} \int \mathrm{d}^{5} x \sqrt{-G}\left(\mathscr{R}-\frac{1}{2}(\partial \phi)^{2}-V(\phi)-\frac{f(\phi)}{4} F_{\mu v} F^{\mu v}\right),
$$

where $F_{\mu \nu}$ is the field strength of the Maxwell field, and $f(\phi)$ determines the coupling of the scalar $\phi$ to $A_{\mu}$. We follow [16] in choosing $f(\phi)=\cosh (12 / 5) / \cosh (6(\phi-2) / 5)$ here, but we have checked that other choices lead to similar results for our observables. Again, we start with the general form (2.4) of the metric. For the case of finite chemical potential we make a similar but slightly simpler ansatz for $A(z)$ than in the case without chemical potential above by setting

$$
A(z)=\log \left(\frac{R}{z}\right), \quad \phi(z)=\sqrt{\frac{3}{2}} \kappa z^{2}, \quad A_{\mu} \mathrm{d} x^{\mu}=\psi(z) \mathrm{d} t .
$$

Here, $\kappa$ is our dimensionful deformation parameter controlling the breaking of conformality. Two different classes of deformed models result again from considering the scalar $\phi$ as a dilaton or not, again called 'string frame' and 'Einstein frame' model, respectively. The equations of motion corresponding to (2.10) are solved for $B, h, V$ and $\psi$, and the solutions can in fact be found in closed form [17]. For given $T$ and $\mu$ there is a maximal deformation $\kappa_{\max }$ for which a black hole solution with these values of $T$ and $\mu$ still exists. It turns out that as a consequence a certain region of small $T$ and $\mu$ is not accessible with metrics of the chosen form. As discussed above, we are for the comparison with the quark-gluon plasma only interested in the region of high $T$ and $\mu$ anyway. Therefore this restriction does not pose a problem for us.

\section{Screening Distance and Running Coupling}

Let us now turn to the screening of a heavy quark-antiquark pair immersed in a plasma. We allow the pair to move with constant velocity $v$ through the plasma. We assume the quarks to be infinitely heavy and work in the probe limit. In the AdS/CFT context this problem has first been discussed in [18] for $\mathscr{N}=4$ SYM theory, and in [19] for the case of the ad hoc deformation that we call $\mathrm{SW}_{T}$. In the AdS description the heavy quark-antiquark is represented by an open string connecting the two quarks and hanging down into the bulk. The ends of the string (i. e. the quarks) 
are located at the boundary $z=0$. The thermal expectation value of a Wegner-Wilson loop of infinite time-extension gives the free energy $F(L)$ of the pair as a function of their spatial distance $L$ through $\langle W(\mathscr{C})\rangle=\exp [-i \mathscr{T} F(L)]$ with the (large) temporal extension $\mathscr{T}$ of the loop. In the (deformed) AdS space the expectation value of the loop is given by $\langle W(\mathscr{C})\rangle \propto \exp \left[i\left(S-S_{0}\right)\right]$, where $S$ is the Nambu-Goto action for the string configuration described above. $S_{0}$ is twice the NambuGoto action for a string hanging down from $z=0$ into the horizon at $z_{h}$. The latter contribution corresponds to the self energy of the quarks, and for moving quarks is closely related to the drag force acting on a single quark pulled through the plasma. The different AdS models discussed in the previous section enter the Nambu-Goto action. In the cases where the scalar $\Phi$ is a dilaton, the metrics entering there are not the ones given above, but need first to be multiplied by a factor involving the dilaton, for example $G_{\alpha \beta}^{s}=\exp (-\sqrt{2 / 3} \Phi) G_{\alpha \beta}^{E}$ for our choice of normalization. This gives rise to the respective metric in the string frame as necessary for the application of the Nambu-Goto action.

One finds that there is always a maximal distance $L_{\max }$ between the quark and antiquark for which a string solution connecting them exists. At larger distances $L>L_{\max }$, no real-valued string solution can be found. For any smaller distance $L<L_{\max }$ there are two string solutions: The one coming closer to the horizon has a higher energy $F(L)$, while the one staying further away from the horizon has a lower energy $F(L)$. The former is thus unstable. In the following we want to concentrate on the maximal possible distance $L_{\max }$ that a bound quark-antiquark pair can have in the plasma. We call this distance the screening distance. ${ }^{1}$ Interestingly, $L_{\max }$ is one of the observables that exhibit a universal behavior for large classes of non-conformal theories.

We have performed the calculation of $L_{\max }$ in all of the models presented in section 2. Specifically, we have studied its dependence on the deformation parameters of the models and on all kinematical parameters, namely the velocity $v=\tanh \eta$ and the orientation angle of the pair with respect to its velocity in the plasma. We first find that $L_{\max }$ is a robust quantity, i. e. it changes only relatively little with changes of the respective conformality-breaking parameters of the different models. For the case of vanishing chemical potential we have plotted in figure 1 the dependence of $L_{\max }$ on the rapidity $\eta$ for several models with typical choices for their respective deformation parameters. The plot shows, for convenience, the dimensionless quantity $\pi T L_{\max }$, and in addition we have divided by the dominant $1 / \sqrt{\cosh \eta}$ behavior of the $\eta$-dependence.

For the case of vanishing chemical potential we have made the following interesting observation. For all given kinematical parameters the screening distance $L_{\max }$ is larger in any of the deformed models than in $\mathscr{N}=4 \mathrm{SYM}$ theory at the same temperature. It is very suggestive to conjecture that this minimality property of $L_{\max }$ in $\mathscr{N}=4 \mathrm{SYM}$ is more general and might even hold in all theories.

The $\eta$-dependence of $L_{\max }$ for finite chemical potential is shown in figure 2 for several models. Generally, the effect of changes in $\mu$ on $L_{\max }$ is smaller than that of changes in $T$. It turns out that for some of the Einstein-frame models and some range of deformation parameter $\kappa$ the screening distance becomes smaller than the corresponding value in $\mathscr{N}=4 \mathrm{SYM}$ at the same $T$ and $\mu$, thus violating the bound just discussed for the case of vanishing $\mu$. However, the violation stays always

\footnotetext{
${ }^{1}$ It must not be confused with the Debye screening length which governs the screening of the quark and antiquark when they are separated far beyond $L_{\max }$.
} 


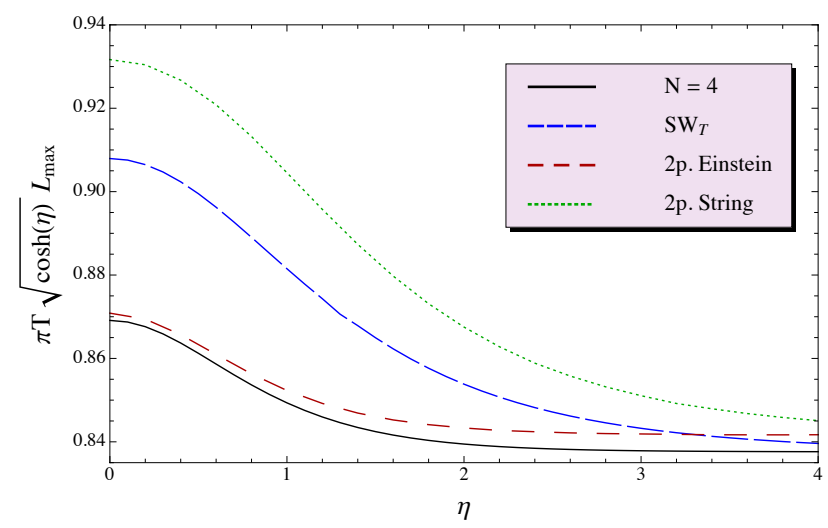

Figure 1: Dimensionless screening distance $\pi T L_{\max } \sqrt{\cosh \eta}$ of a $Q \bar{Q}$ pair as a function of rapidity $\eta$ for different models with vanishing chemical potential.

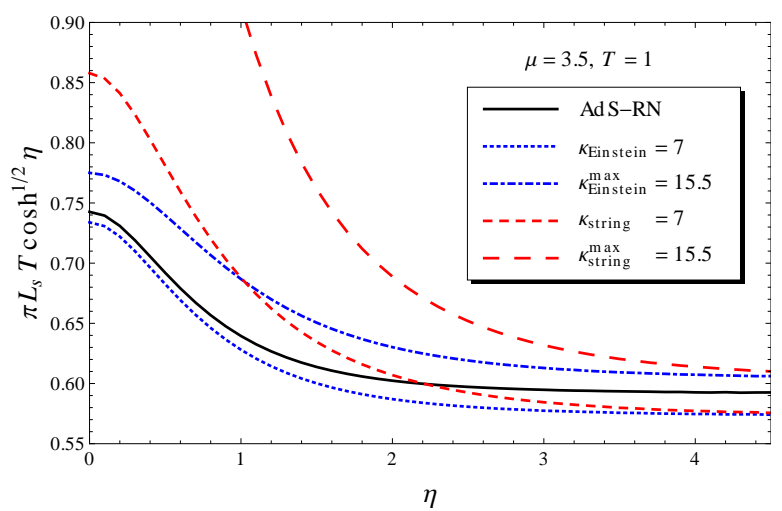

Figure 2: Dimensionless screening distance $\pi T L_{\max } \sqrt{\cosh \eta}$ of a $Q \bar{Q}$ pair as a function of rapidity $\eta$ for different models with finite chemical potential.

small. It is conceivable that an improved bound can be found for finite $\mu$.

Let us now turn to the running coupling in our AdS models. It can be defined in terms of the free energy $F(L)$ of the heavy quark-antiquark pair as

$$
\alpha_{q q}(r=L, T, \mu) \equiv \frac{3}{4} L^{2} \frac{\mathrm{d} F(L, T, \mu)}{\mathrm{d} L} .
$$

The normalization, here chosen as in QCD, is somewhat ambiguous as we do in general not know the Casimir factors of the theories dual to our AdS models. The normalization of $\alpha_{q q}$ further depends on the 't Hooft coupling of the theory. We therefore treat the normalization as a free parameter in our calculation. We have computed $\alpha_{q q}$ for various AdS models at vanishing and at finite chemical potential $[23,17]$. As an example of the $\mu=0$ case we show in figure 3 the running coupling as obtained in the $\mathrm{SW}_{T}$ model for various temperatures. For $T_{c}$ we have chosen $176 \mathrm{MeV}$ here. The curves end at the screening distance $L_{\max }$ of the respective temperature. We observe the universal property that $\alpha_{q q}$ develops a maximum before falling steeply close to $L_{\max }$. This holds for all non-conformal models, including those with finite chemical potential. Also for this observable, the effect of changing $T$ is considerably stronger than the effect of changing $\mu$. 


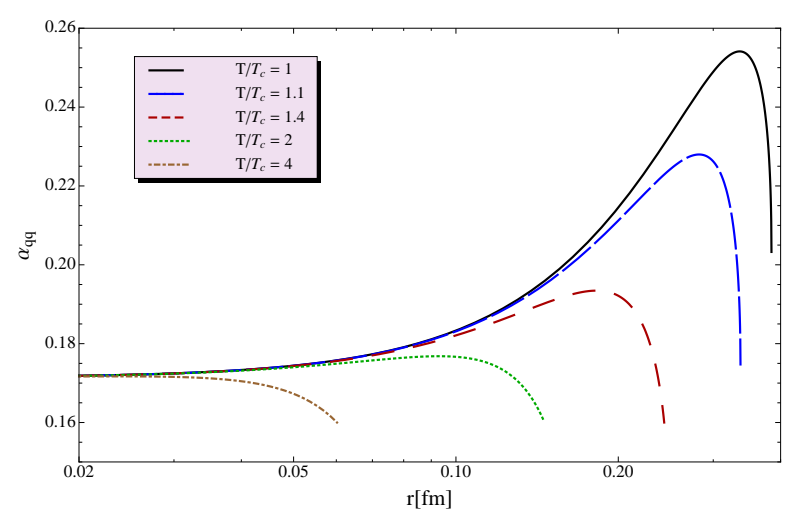

Figure 3: Running coupling $\alpha_{q q}$ as function of the distance $r$ for the temperatures $T / T_{c}, T / T_{c}=1,1.1,1.4,2$ and 4 and for a fixed deformation $c / T^{2}$ in the $\mathrm{SW}_{T}$ model.

Let us finally point out that in the 2-parameter model at finite $T$ (but vanishing $\mu$ ) one can adjust the free parameters such that $\alpha_{q q}$ comes close to the corresponding QCD lattice data [20] over a sizeable range of temperatures.

\section{Accelerated Motion}

Let us now turn to the accelerated motion of a heavy quark in the plasma. In the AdS/CFT framework, the simplest motion of this kind is a circular motion. The quark's energy loss due to radiation can, from a theoretical perspective, provide interesting information about the plasma, although a continuous circular motion of a quark is clearly not a realistic situation in any experiment.

Let us consider a quark moving on a circle of radius $R_{0}$ with constant angular velocity $\omega$. For $\mathscr{N}=4 \mathrm{SYM}$ this situation has been considered for $T=0$ and $T \neq 0$ in [21] and [22], respectively. We have calculated the energy loss in the non-conformal metrics of section 2, both at $\mu=0$ [23] and at $\mu \neq 0$ [24]. Typical configurations of the open string hanging down from the rotating quark into the bulk are shown in figure 4. The left panel corresponds to pure $\mathrm{AdS}_{5}$ space at $T=0$ and shows the string for two different angular velocities. For $T=0$ there is no black hole horizon and consequently the string spirals out to infinite radii deep in the bulk. The situation is different at finite $T$ where the motion of the string is limited in the holographic direction by the horizon, such that the string can reach only a finite radius, see right panel.

We find that the string solution for the non-conformal models is always very close to the one for $\mathscr{N}=4 \mathrm{SYM}$ in the region close to the boundary, i.e. for small $z$. One can show that only the part of the string close to the boundary determines the energy radiation off the quark. Therefore, the radiation pattern in the non-conformal models is generally very similar to that of $\mathscr{N}=4 \mathrm{SYM}$. This holds both at vanishing and finite chemical potential.

Finally, we observe that the rotating quark loses more energy at higher $T$ and $\mu$. Again, the effect of changing $T$ is considerably larger than the effect of changing $\mu$, as is seen in figure 5 for the example of $\mathscr{N}=4 \mathrm{SYM}$ (without deformation) corresponding to an AdS-RN metric. 

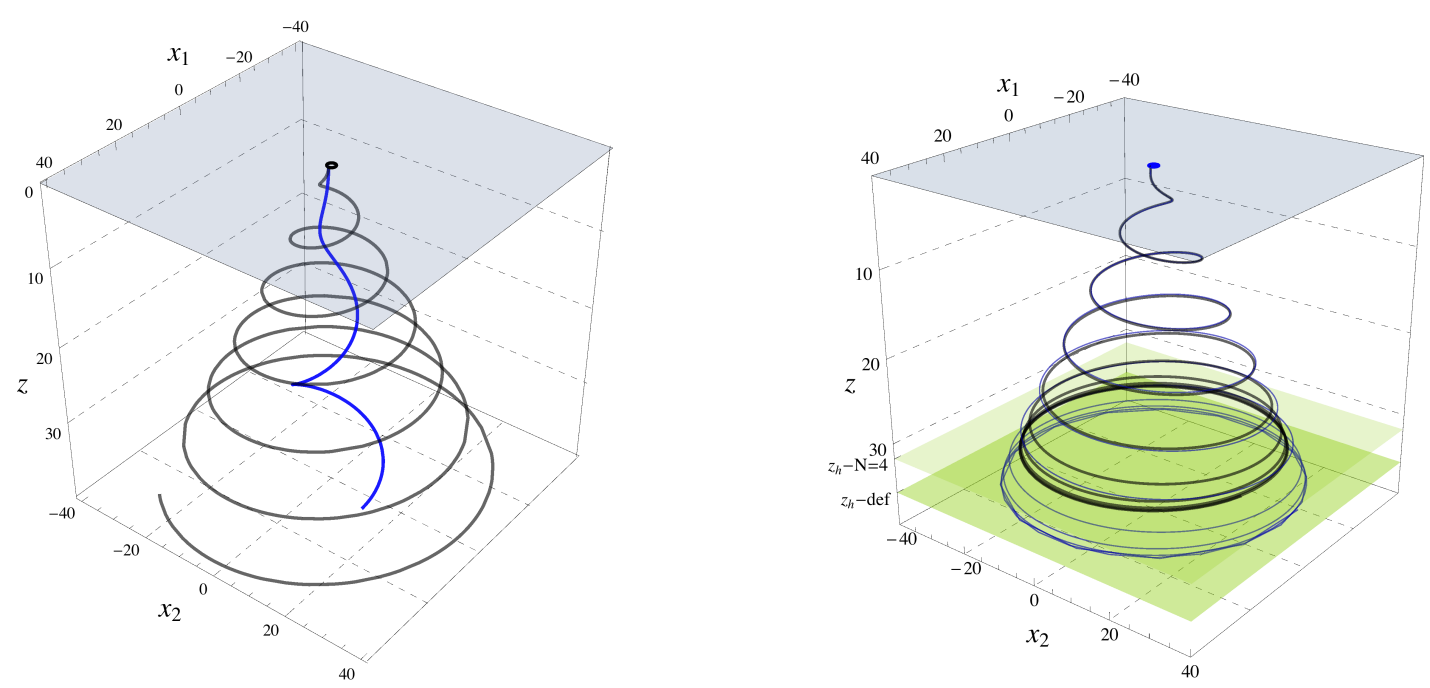

Figure 4: Left: String configuration in pure $\operatorname{AdS}_{5}(\mathscr{N}=4 \mathrm{SYM}$ at $T=0)$ with radius $R_{0}=1$ and angular velocity $\omega=0.3$ (blue) and 0.7 (black). The quark rotates on the small black circle at the top. Right: String configuration in 2-parameter string-frame model (blue) for typical values of $\alpha$ and $c$ together with the corresponding $\mathscr{N}=4$ case (black) at $T=0.01, R_{0}=1$ and $\omega=0.7$ (in dimensionless units).

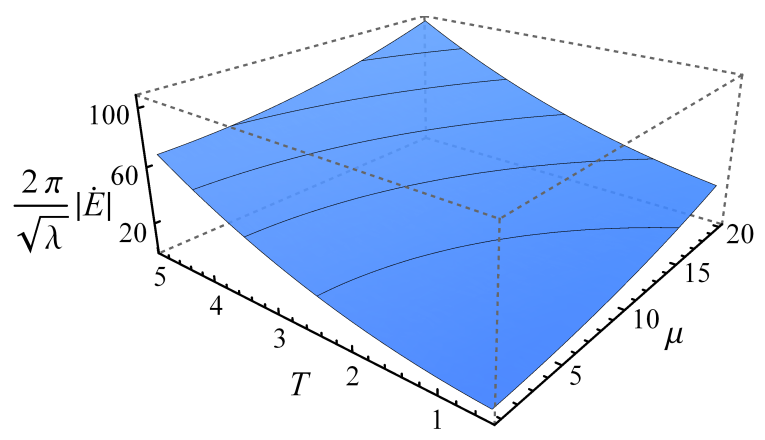

Figure 5: Energy loss per unit time of a rotating quark in $\mathscr{N}=4 \mathrm{SYM}$ theory as a function of temperature $T$ and chemical potential $\mu$, both measured in the same arbitrary units. The quark moves with angular velocity $\omega=1 / 2$ on a circle of radius $R_{0}=1$ in these units.

\section{Summary}

We have studied several observables related to heavy quarks in strongly coupled plasmas in large classes of theories, using the framework of the AdS/CFT duality. The screening distance of a heavy quark-antiquark pair, the running coupling, as well as the energy loss of a heavy quark in circular motion have been studied. They are found to be robust under non-conformal deformations of the holographic theories and exhibit universal behavior. This universal behavior might be a sign of general strong-coupling dynamics, indicating a potential relevance of our findings for the quark-gluon plasma observed in heavy-ion collisions.

\section{Acknowledgments}

The work of K. S. was supported in part by the International Max Planck Research School for 
Precision Tests of Fundamental Symmetries. This work was supported by the Alliance Program of the Helmholtz Association (HA216/EMMI).

\section{References}

[1] J. M. Maldacena, Adv. Theor. Math. Phys. 2, (1998) 231 [Int. J. Theor. Phys. 38, (1999) 1113] [arXiv:hep-th/9711200].

[2] S. S. Gubser, I. R. Klebanov and A. M. Polyakov, Phys. Lett. B 428 (1998) 105 [arXiv:hep-th/9802109].

[3] E. Witten, Adv. Theor. Math. Phys. 2, (1998) 253 [arXiv:hep-th/9802150].

[4] J. Casalderrey-Solana, H. Liu, D. Mateos, K. Rajagopal and U. A. Wiedemann, Gauge/String Duality, Hot QCD and Heavy Ion Collisions, Cambridge University Press 2014, Cambridge, UK [arXiv:1101.0618 [hep-th]].

[5] O. DeWolfe, S. S. Gubser, C. Rosen and D. Teaney, Prog. Part. Nucl. Phys. 75 (2014) 86 [arXiv:1304.7794 [hep-th]].

[6] U. Gursoy, E. Kiritsis, L. Mazzanti, G. Michalogiorgakis and F. Nitti, Lect. Notes Phys. 828 (2011) 79 [arXiv:1006.5461 [hep-th]].

[7] G. Policastro, D. T. Son and A. O. Starinets, Phys. Rev. Lett. 87 (2001) 081601 [arXiv:hep-th/0104066].

[8] P. Kovtun, D. T. Son and A. O. Starinets, Phys. Rev. Lett. 94 (2005) 111601 [arXiv:hep-th/0405231].

[9] O. Andreev and V. I. Zakharov, Phys. Rev. D 74 (2006) 025023 [hep-ph/0604204].

[10] K. Kajantie, T. Tahkokallio and J. T. Yee, JHEP 0701, (2007) 019 [arXiv:hep-ph/0609254].

[11] A. Karch, E. Katz, D. T. Son and M. A. Stephanov, Phys. Rev. D 74 (2006) 015005 [hep-ph/0602229].

[12] C. Ewerz, L. Lin, A. Samberg, in preparation

[13] S. S. Gubser and A. Nellore, Phys. Rev. D 78 (2008) 086007 [arXiv:0804.0434 [hep-th]].

[14] O. DeWolfe and C. Rosen, JHEP 0907 (2009) 022 [arXiv:0903.1458 [hep-th]].

[15] P. Colangelo, F. Giannuzzi and S. Nicotri, Phys. Rev. D 83 (2011) 035015 [arXiv:1008.3116 [hep-ph]].

[16] O. DeWolfe, S. S. Gubser and C. Rosen, Phys. Rev. D 83 (2011) 086005 [arXiv:1012.1864 [hep-th]].

[17] A. Samberg, Diploma thesis, Heidelberg University, 2012; C. Ewerz, A. Samberg, in preparation

[18] H. Liu, K. Rajagopal and U. A. Wiedemann, Phys. Rev. Lett. 98 (2007) 182301 [arXiv:hep-ph/0607062].

[19] H. Liu, K. Rajagopal and Y. Shi, JHEP 0808, (2008) 048 [arXiv:0803.3214 [hep-ph]].

[20] O. Kaczmarek, F. Karsch, F. Zantow and P. Petreczky, Phys. Rev. D 70 (2004) 074505 [Erratum-ibid. D 72 (2005) 059903] [arXiv:hep-lat/0406036].

[21] C. Athanasiou, P. M. Chesler, H. Liu, D. Nickel and K. Rajagopal, Phys. Rev. D 81 (2010) 126001 [Erratum-ibid. D 84 (2011) 069901] [arXiv:1001.3880 [hep-th]].

[22] K. B. Fadafan, H. Liu, K. Rajagopal and U. A. Wiedemann, Eur. Phys. J. C 61 (2009) 553 [arXiv:0809.2869 [hep-ph]].

[23] K. Schade, PhD thesis, Heidelberg University, 2012; C. Ewerz, K. Schade, in preparation

[24] L. Lin, MSc thesis, Heidelberg University, 2013; C. Ewerz, L. Lin, in preparation 